\title{
Antibacterial and Dye Degradation Activity of Green Synthesized Iron Nanoparticles
}

\author{
Arpita Roy ${ }^{(D},{ }^{1}$ Vishwajeet Singh, ${ }^{1}$ Sukriti Sharma, ${ }^{1}$ Daoud Ali, ${ }^{2}$ Abul Kalam Azad $\left(\mathbb{D},{ }^{3}\right.$ \\ Gokhlesh Kumar, ${ }^{4}$ and Talha Bin Emran ${ }^{5}{ }^{5}$
}

${ }^{1}$ Department of Biotechnology, School of Engineering \& Technology, Sharda University, Greater Noida, India

${ }^{2}$ Department of Zoology, College of Science, King Saud University, PO Box 2455, Riyadh 11451, Saudi Arabia

${ }^{3}$ Pharmaceutical Technology Unit, Faculty of Pharmacy, AIMST University, Bedong, 08100 Kedah, Malaysia

${ }^{4}$ Clinical Division of Fish Medicine, University of Veterinary Medicine Vienna, 1210 Vienna, Austria

${ }^{5}$ Department of Pharmacy, BGC Trust University Bangladesh, Chittagong 4381, Bangladesh

Correspondence should be addressed to Arpita Roy; arbt2014@gmail.com and Talha Bin Emran; talhabmb@gmail.com

Received 10 December 2021; Accepted 20 December 2021; Published 17 January 2022

Academic Editor: Palanivel Velmurugan

Copyright (C) 2022 Arpita Roy et al. This is an open access article distributed under the Creative Commons Attribution License, which permits unrestricted use, distribution, and reproduction in any medium, provided the original work is properly cited.

\begin{abstract}
Nanoparticles have a wide range of applications in various fields such as cosmetics, pharmaceuticals, and agrochemicals. Synthesis of nanoparticles using plant extract is a very efficient, cost-effective, useful, and environmentally friendly method. A plant extract acts as a reducing agent in the formation of nanoparticles. Catharanthus roseus is one of the potential plants for biosynthesis of nanoparticles due to its easy availability. In the present study, the Catharanthus roseus plant extract was used to synthesize iron nanoparticles. UV-vis spectroscopy, DLS, and FTIR were performed for characterization of synthesized nanoparticles. Further antibacterial and dye degrading properties of synthesized iron nanoparticles have been investigated. It was found that Catharanthus roseus-synthesized iron nanoparticles showed antibacterial activity against E. coli and dye degradation activity against methyl orange dye.
\end{abstract}

\section{Introduction}

Nanotechnology has significant applications in various fields including agriculture, medicine, energy, climate, chemistry, heavy industry, and consumer goods [1-5]. They have a high surface area to volume ratio, which is the most important aspect that explains why these nanomaterials are so commonly used [6,7]. Various approaches, including physical and chemical methods, have been used to synthesize NPs $[8$, 9]. Physical and chemical methods of synthesis possess certain drawback such as high energy consumption and toxic chemical utilization [4]. Most studies have used sodium borohydride as a reducing agent to reduce ferrous (Fe (II)) or ferric (Fe (III)) salts. Nanoparticles are easily synthesized using $\mathrm{NaBH}_{4}$, but it is potentially hazardous for environment [10]. Therefore, an alternative method for synthesis is in high demand. A green method for synthesis of iron nanoparticles (FeNPs) uses biological entities such as fungi, bacteria, and plants which have been utilized in the present time. Plant-mediated approaches are straightforward, cost-effective, and environmentally sustainable [6]. A plant extract contains bioactive compounds such as polyphenols, alkaloids, steroids, flavonoids, and terpenoids [11-13] which act as both reducing and stabilizing agents at the same time. FeNPs have been synthesized using a number of plants, including Peltophorum pterocarpum, Eriobotrya japonica, Mentha piperita, and Moringa oleifera [14-17].

Catharanthus roseus commonly known as graveyard vine, bright eyes, Madagascar periwinkle, cape periwinkle, etc. is a flowering plant that belongs to the Apocynaceae family. Pentamerous flowers are present with different colors such as pink, purple, white, peach, scarlet, and red. It is an important medicinal plant which is native to Madagascar but cultivated in various parts of the world [18]. It contains various bioactive compounds such as vinblastine, vincristine, reserpine, and vinpocetine which possess several 
pharmaceutical activities such as antibacterial, antioxidant, antidiabetic, antifungal, and anticancer properties [19]. Catharanthus roseus has been utilized for synthesis of various nanoparticles which includes silver, gold, copper oxide, iron, etc. Iron nanoparticles have been shown to have various environmental remediation potentials. Water and wastewater toxins are currently being remedied using nanoscale materials such as nanoadsorbents, nanofiltration, nanocatalysts, and nanobiocides such as metal nanoparticles. Iron nanoparticles are one of these metallic nanoparticles with promising environmental pollution-fighting properties [20]. KatataSeru et al. [16] reported antibacterial activity of biosynthesized FeNPs. The aim of this study is to synthesize iron nanoparticles from C. roseus, and further FeNP formation was confirmed using different characterization methods such as UV-visible spectroscopy, FTIR, and DLS. Bactericidal activity and dye degradation activity of synthesized FeNPs were also evaluated.

\section{Materials and Methods}

Nutrient broth (NB), nutrient agar (NA), streptomycin disc, ferrous sulphate salt, and methyl orange were bought from Fisher Scientific.

2.1. Plant Extract Preparation. Leaves of Catharanthus roseus were collected from Sharda University. Then, leaves were washed with detergent and then with distilled water 2-3 times to remove detergent from its surface. After washing properly, leaves were dried in an oven at $60^{\circ} \mathrm{C}$ for $48 \mathrm{hrs}$. Dried leaves were ground to powdered form. Then, $1 \mathrm{~g}$ of plant extract powder was added into $50 \mathrm{ml}$ of distilled water (DW) and boiled for $20 \mathrm{~min}$. Then, it was filtered with Whatman no. 1 filter paper and stored at $4^{\circ} \mathrm{C}$ for further use.

2.2. Optimization of Synthesis of $\mathrm{FeSO}_{4}$ and Their Characterization. To synthesize FeNPs, ferrous sulphate salt $\left(\mathrm{FeSO}_{4}\right)$ was used. To make a $150 \mathrm{mM}$ ferrous sulphate solution, $4.3 \mathrm{~g}$ ferrous sulphate was mixed with $100 \mathrm{ml}$ distilled water. The plant extract was added to the ferrous sulphate in $1: 9$, and $1 \mathrm{ml}$ of the plant extract and $9 \mathrm{ml}$ of the ferrous sulphate are mixed for the synthesis of copper nanoparticles. Different concentrations of $\mathrm{FeSO}_{4}(1,10,50$, and $100 \mathrm{mM})$ and plant extract $(1,2,3,4$, and $5 \mathrm{ml})$ were used for nanoparticle synthesis. A UV-vis spectrophotometer was used to detect the reduction of $\mathrm{Fe}$ ions into FeNPs, which resulted in a yellow color formation. Iron nanoparticles were detected in the wavelength range of $200-300 \mathrm{~nm}$. Centrifugation at $4500 \mathrm{rpm}$ for 15 minutes purified the nanoparticles. FTIR was used to determine iron ion reduction and capping of nanoparticles. The size and size distribution of molecules were measured using DLS.

2.3. Antibacterial Activity. Synthesized copper nanoparticles were evaluated against E. coli. $200 \mathrm{ml}$ of NA and NB was prepared. $100 \mathrm{ml}$ of NB was used for the growth of the bacteria. The disk diffusion method was used to observe antibacterial activity. The culture was then spread uniformly on agar plates with the help of a swab. Various concentrations of FeNPs were added on a sterile disk using a pipette. A

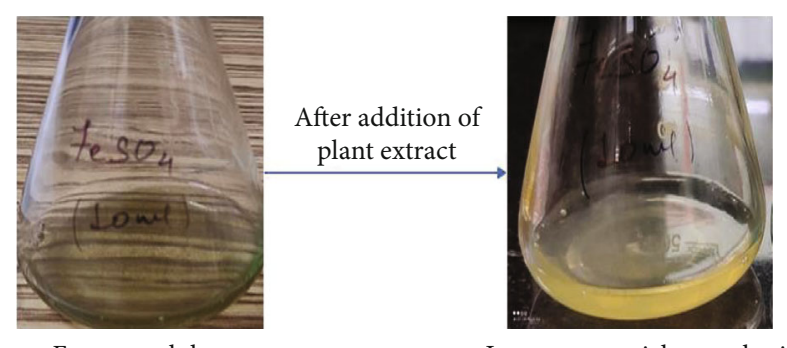

Ferrous sulphate

Figure 1: Synthesis of FeNPs.

streptomycin-loaded disk was used as the positive control and disk without NPs used as the negative control. Plates were then incubated at $37^{\circ} \mathrm{C}$ overnight for $48 \mathrm{hrs}$, and the zone of inhibition was observed.

2.4. Dye Degradation Activity. The photocatalytic dye degradation potential of FeNPs was evaluated for methyl orange. In $3 \mathrm{ml}$ of DW, $1 \mathrm{ml}$ of $1 \mathrm{mM}$ concentration dye and $1 \mathrm{ml}$ of FeNPs were mixed to make the total volume up to $5 \mathrm{ml}$. Then, the solution was kept in sunlight. After this, a UVvis spectrophotometer was used to measure the change in intensity of color in the range $200-800 \mathrm{~nm}$.

\section{Results and Discussion}

There are several bioactive compounds present in the plant extract. These compounds react with $\mathrm{FeSO}_{4}$ to synthesize FeNPs. The color of the $\mathrm{FeSO}_{4}$ solution was bluish green before adding the plant extract. There was a change in color from bluish green to yellow (Figure 1). This suggests that the plant extract aids in the formation of nanoparticles. Akhbari et al. [15] reported that there was some visible change in the reaction mixture that contains the iron sulfate salt and plant extract of Mentha piperita from yellow to dark brown which shows synthesis of FeNPs.

3.1. Effect of Different Concentrations of Ferrous Sulfate. FeNP formation was detected using UV-vis analysis. Change in color was observed due to the reduction of iron ions from ferrous sulphate to FeNPs. FeNP peak was observed at around $240 \mathrm{~nm}$. Saini et al. [21] reported absorbance peak of FeNPs at around $210-260 \mathrm{~nm}$ region. Ebrahiminezhad et al. [22] reported that UV-vis spectroscopy of biosynthesized FeNP nanoparticles from Urtica dioica was in the range of $216-265 \mathrm{~nm}$. Akhbari et al. [15] reported that at wavelengths around 216 and $284 \mathrm{~nm}$, two different absorption peaks of FeNPs were observed.

Various concentrations of ferrous sulphate were investigated for optimum synthesis of FeNPs. As the concentration of ferrous sulphate rises, there was an increase in FeNP synthesis. For all the concentrations, peak was about at $230 \mathrm{~nm}$ (Figure 2). The rate of nanoparticle synthesis was determined by ferrous sulphate concentration. At higher concentrations, particle size grows larger, and spectrum strength grows as well.

Effect of different plant extract concentrations (1-5 ml) on the synthesis of FeNPs was also investigated. UV-vis 


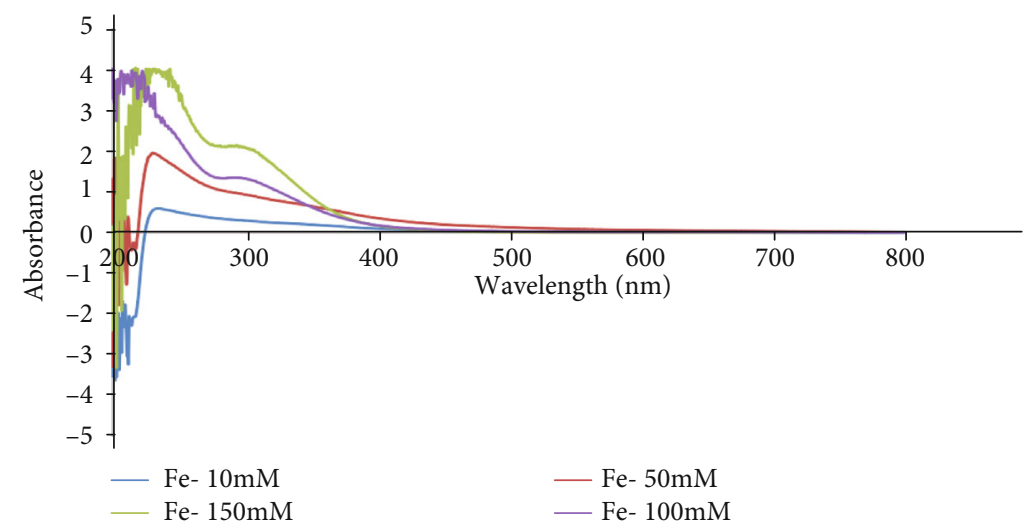

Figure 2: UV-vis spectra of FeNPs at different concentrations of $\mathrm{FeSO}_{4}$.

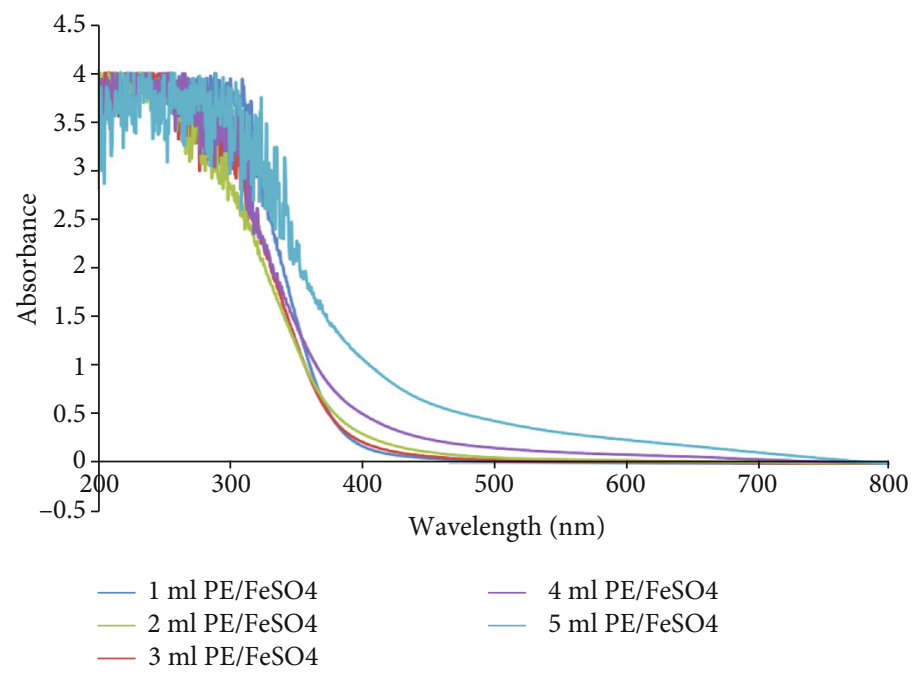

FIgURE 3: UV-vis spectra of FeNPs at various plant extract concentrations.

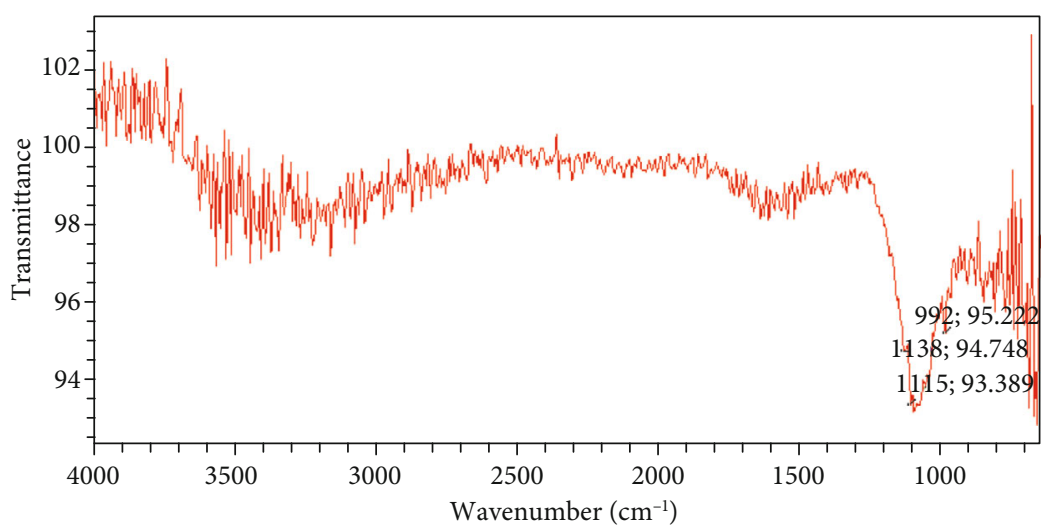

FIGURE 4: FTIR analysis of FeNPs.

spectra showed that maximum nanoparticle synthesis was obtained in the case of $5 \mathrm{ml}$ of $C$. roseus plant extract solution (Figure 3 ). As a result, this confirms that as the concentration of the plant extract increased, nanoparticles synthesis also increased. Vitta et al. [23] reported synthesis of iron nanoparticles from Eucalyptus robusta, and they found that
$1: 1$ proportion of salt concentration to the leaf extract was optimum for synthesis of nanoparticles.

3.2. FTIR Analysis. The presence of different functional groups in the synthesized FeNPs was identified by using FT-IR spectra (Figure 4). At a range of $4000-650 \mathrm{~cm}^{-1}$, 
TABLE 1: FT-IR analysis of iron nanoparticles.

\begin{tabular}{|c|c|c|}
\hline Vibrational peak $\left(\mathrm{cm}^{-1}\right)$ & Vibrational frequencies & Reference \\
\hline $1115 \mathrm{~cm}^{-1}$ & $\begin{array}{c}\mathrm{C}=\mathrm{O} \text { indicates the presence of aldehydes and ketones which means the occurrence of } \\
\text { phenolic acid and terpenoid }\end{array}$ & {$[25]$} \\
\hline $1135 \mathrm{~cm}^{-1}$ & $\begin{array}{c}\mathrm{C}=\mathrm{O} \text { indicates the presence of aldehydes and ketones which means the occurrence of } \\
\text { phenolic acid and terpenoid }\end{array}$ & {$[25]$} \\
\hline $992 \mathrm{~cm}^{-1}$ & $\mathrm{C}-\mathrm{N}$ indicated the presence of phenols and aliphatic amines & {$[22]$} \\
\hline
\end{tabular}

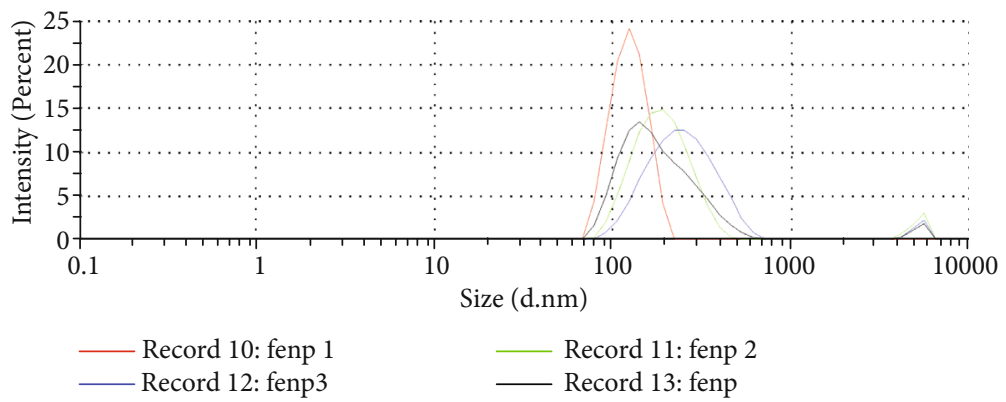

FIGURE 5: DLS analysis of FeNPs.

measurement was made with a resolution of $4 \mathrm{~cm}^{-1}$. FTIR spectra showed three major peaks in the range of $800-$ $1200 \mathrm{~cm}^{-1}$ (Table 1). Shahwan et al. [24] reported that different functional groups were responsible for FeNP synthesis. Wei et al. [25] reported the presence of peak at 3292.84, $2927.78,1638.55,1350.99$, and $1026.60 \mathrm{~cm}^{-1}$ in the case of iron nanoparticles synthesized using the peel of Citrus maxima. Akhbari et al. [15] confirms the presence of some functional groups which shows that the extracts contain aliphatic amines, phenols, and organic acids that could serve as stabilizing and reducing agents during FeNP synthesis. Karpagavinayagam and Vedhi [26] reported the presence of peak at $3422,2923,2853,1630,618$, and $467 \mathrm{~cm}^{-1}$ in the case of iron nanoparticles synthesized using the flower extract of Avicennia marina. Dash et al. [17] reported the presence of peak at $3700,3400,2918,2360,1000,800,717,623,557$, and $443 \mathrm{~cm}^{-1}$ in the case of iron nanoparticles synthesized using Peltophorum pterocarpum.

3.3. DLS Analysis. The most popular application of DLS is to examine nanoparticles. The technique is suitable for particles in submicron, but it can also be used for calculating the particles smaller than nanometer. The spontaneous variations in the strength of light dispersed from any solution or any suspension may be used to calculate particle size. In this study, zeta potential was found to be $7.75 \mathrm{mV}$, with a zeta deviation of $5.77 \mathrm{mV}$ as shown in (Figure 5). The polydispersity index of FeNPs is 0.411. Green synthesized FeNPs had an average particle size distribution of $290.1 \mathrm{~nm}$. Since biomolecules or a layer of water covering the NP's surface is included in the calculation, FeNPs have a larger scale.

3.4. Antibacterial Activity. Antibacterial activity of FeNPs synthesized from Catharanthus roseus was tested against a gram-negative bacterial culture of E. coli. Results showed the potential antibacterial activity of these FeNPs against $E$. coli after 24 hours of incubation. It was observed that the

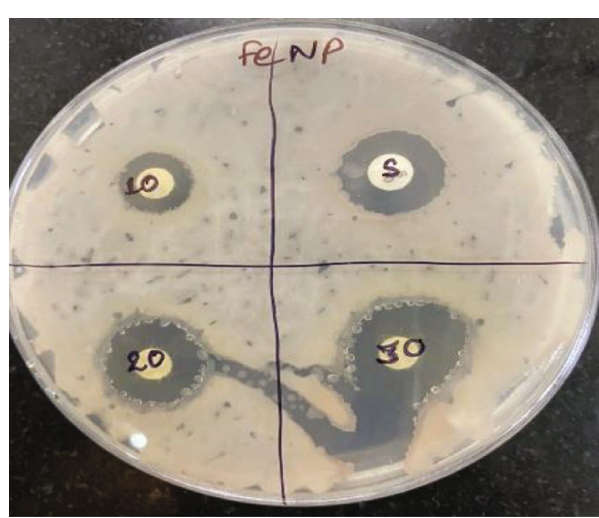

FIgURE 6: Antibacterial activity of FeNP against E. coli.

zone of inhibition increases with the increase in amount of FeNPs (Figure 6). The minimum zone of inhibition was found in 10 microliters of FeNP solution, and 30 microliters of solution shows the maximum zone of inhibition. The zone of $30 \mu \mathrm{l}$ of NP was the maximum with $15 \mathrm{~mm} \pm 0.5$ of diameter followed by $20 \mu \mathrm{l}$ with $12 \mathrm{~mm} \pm 0.5$ and then $10 \mu \mathrm{l}$ with $9 \mathrm{~mm} \pm 1$, and the least was of streptomycin with $11 \mathrm{~mm} \pm 0.1$ of diameter. Senthil and Ramesh [27] reported the antibacterial activity of Tridax procumbens-synthesized iron oxide against the gram-negative bacteria. Dash et al. [17] reported the antibacterial activity of magnetite nanoparticle synthesis using the pod extract of Peltophorum pterocarpum against E. coli. A maximum of $16 \mathrm{~mm}$ and $20 \mathrm{~mm}$ of the zone of inhibition was obtained for S. epidermis and E. coli, respectively.

3.5. Dye Degradation Activity. One of the efficient applications of FeNPs is dye degradation. Methyl orange (MO) is a harmful and toxic dye, which is found in general food 


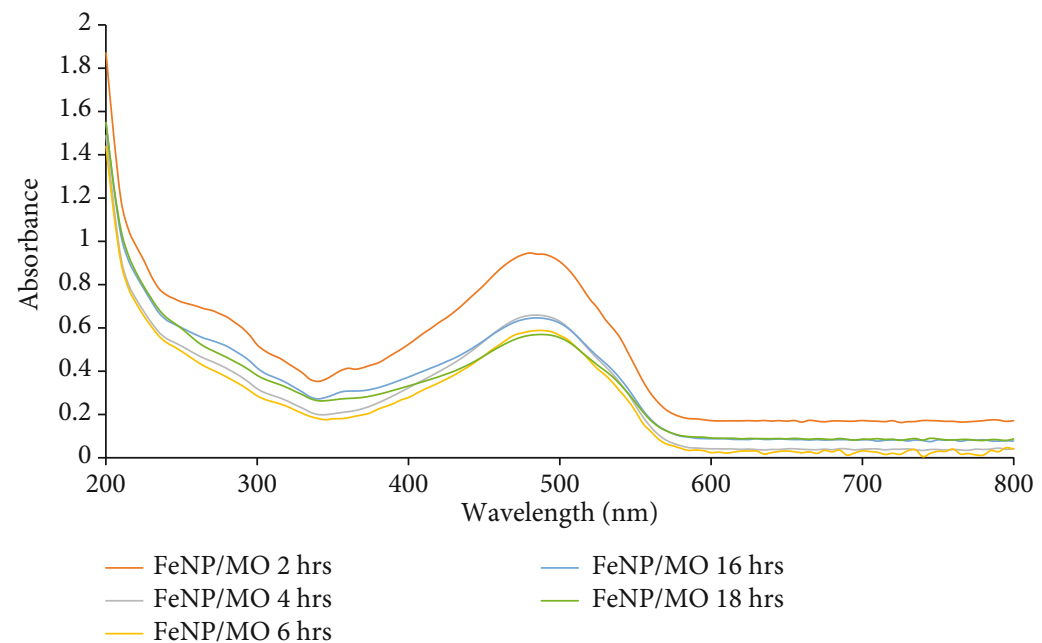

Figure 7: Photocatalytic dye degradation of methyl orange using FeNPs.

and textile industries; it is carcinogenic. Efficiency of the synthesized FeNPs for photocatalytic degradation was examined using methyl orange dye. UV-vis spectra of MO in water generally appear at $500 \mathrm{~nm}$. Within 2 hours, degradation of dye using nanoparticles was observed at uniform time intervals. Dye absorbance declines with time, degradation process reduces as time passes, and this may be due to the binding of degraded materials to the nanoparticle surface (Figure 7). Maximum 50\% degradation of dye has been observed, and complete degradation of dye was not observed. Bishnoi et al. [28] reported methylene blue degradation using Cynometra ramiflora-derived iron nanoparticles. Kouhbanani et al. [29] reported removal of methyl orange dyes using Artemisia vulgaris-derived iron nanoparticles. Radini et al. [30] reported degradation of MO by a photocatalytic method using Trigonella foenum-graecumderived iron nanoparticles.

\section{Conclusion}

The biological method of nanoparticles synthesis is one of the potential methods which has gained lot of attention. It produces novel materials that are cost-effective and ecofriendly and shows applications in the different areas. In this study, plant extract solution of Catharanthus roseus was used for FeNP synthesis. The plant extract works as a reducing agent and provides stability to nanoparticles. Synthesized FeNPs showed potential antibacterial activity against E. coli. Photocatalytic degradation using synthesized FeNPs showed around $50 \%$ degradation of methyl orange. So, it was concluded that the $C$. roseus can possibly be used for the synthesis of FeNPs.

\section{Data Availability}

The data used to support the findings of this study are included within the article.

\section{Conflicts of Interest}

The authors declare that they have no conflict of interest.

\section{Authors' Contributions}

AR was responsible for the conceptualization. AR, VS, SS, and DA were responsible for the methodology. AR, VS, SS, DA, AKA, GK, and TBE wrote and corrected the manuscript. DA and AR were responsible for the funding acquisition.

\section{Acknowledgments}

This work was funded by Researchers Supporting Project number RSP-2021/165, King Saud University, Riyadh, Saudi Arabia. Also, the author (AR) is grateful to Sharda University for Seed Fund-4 2001 (SUSF2001/12).

\section{References}

[1] A. Roy and N. Bharadvaja, "Silver nanoparticles synthesis from a pharmaceutically important medicinal plant Plumbago zeylanica," MOJ Bioequivalence \& Bioavailability (MOJBB), vol. 3, no. 5, p. 46, 2017.

[2] A. Roy and N. Bharadvaja, "Qualitative analysis of phytocompounds and synthesis of silver nanoparticles from Centella asiatica," Innovative Techniques in Agriculture, vol. 1, no. 2, pp. 88-95, 2017.

[3] A. Roy and N. Bharadvaja, "Silver nanoparticle synthesis from Plumbago zeylanica and its dye degradation activity," Bioinspired, Biomimetic and Nanobiomaterials, vol. 8, no. 2, pp. 130-140, 2019.

[4] A. Roy, A. Elzaki, V. Tirth et al., "Biological synthesis of nanocatalysts and their applications," Catalysts, vol. 11, no. 12, p. 1494, 2021.

[5] A. Roy, "Plant derived silver nanoparticles and their therapeutic applications," Current Pharmaceutical Biotechnology, vol. 22, no. 14, pp. 1834-1847, 2021. 
[6] S. Raina, A. Roy, and N. Bharadvaja, "Degradation of dyes using biologically synthesized silver and copper nanoparticles," Environmental Nanotechnology, Monitoring \& Management, vol. 13, article 100278, 2020.

[7] P. Nagore, S. Ghotekar, K. Mane, A. Ghoti, M. Bilal, and A. Roy, "Structural properties and antimicrobial activities of Polyalthia longifolia leaf extract-mediated $\mathrm{CuO}$ nanoparticles," BioNanoScience, vol. 11, no. 2, pp. 579-589, 2021.

[8] A. Roy, "Synthesis of silver nanoparticles from medicinal plants and its biological application: a review," Research \& Reviews in BioSciences, vol. 12, no. 4, p. 138, 2017.

[9] H. M. Ahmed, A. Roy, M. Wahab et al., "Applications of nanomaterials in agrifood and pharmaceutical industry," Journal of Nanomaterials, vol. 2021, Article ID 1472096, 10 pages, 2021.

[10] B. Desalegn, M. Megharaj, Z. Chen, and R. Naidu, "Green synthesis of zero valent iron nanoparticle using mango peel extract and surface characterization using XPS and GC-MS," Heliyon, vol. 5, no. 5, article e01750, 2019.

[11] A. Roy, "A review on the alkaloids an important therapeutic compound from plants," IJPB, vol. 3, no. 2, pp. 1-9, 2017.

[12] A. Roy, N. Jauhari, and N. Bharadvaja, "Medicinal plants as a potential source of chemopreventive agents," in Anticancer Plants: Natural Products and Biotechnological Implements, pp. 109-139, Springer, Singapore, 2018.

[13] A. Roy, T. Attre, N. Bharadvaja, A. Tiezzi, and T. M. Karpiński, "Anticancer agent from medicinal plants: a review," New Apects in Medicinal Plants and Pharmacognosy, vol. 1, p. 54, 2017.

[14] E. S. Önal, T. Yatkin, M. Ergüt, and A. Özer, "Green synthesis of iron nanoparticles by aqueous extract of Eriobotrya japonica leaves as a heterogeneous Fenton-like catalyst: degradation of basic red 46," International Journal Of Chemical Engineering And Applications, vol. 8, no. 5, pp. 327-333, 2017.

[15] M. Akhbari, R. Hajiaghaee, R. Ghafarzadegan, S. Hamedi, and M. Yaghoobi, "Process optimisation for green synthesis of zero-valent iron nanoparticles using Mentha piperita," IET Nanobiotechnology, vol. 13, no. 2, pp. 160-169, 2018.

[16] L. Katata-Seru, T. Moremedi, O. S. Aremu, and I. Bahadur, "Green synthesis of iron nanoparticles using Moringa oleifera extracts and their applications: Removal of nitrate from water and antibacterial activity against_Escherichia coli_," Journal of Molecular Liquids, vol. 256, pp. 296-304, 2018.

[17] A. Dash, M. T. Ahmed, and R. Selvaraj, "Mesoporous magnetite nanoparticles synthesis using the Peltophorum pterocarpum pod extract, their antibacterial efficacy against pathogens and ability to remove a pollutant dye," Journal of Molecular Structure, vol. 1178, pp. 268-273, 2019.

[18] A. Das, S. Sarkar, S. Bhattacharyya, and S. Gantait, "Biotechnological advancements in Catharanthus roseus (L.) G. Don," Applied Microbiology and Biotechnology, vol. 104, no. 11, pp. 4811-4835, 2020.

[19] Y. Yulizar, J. Gunlazuardi, D. O. B. Apriandanu, and T. W. W. Syahfitri, "CuO-modified $\mathrm{CoTiO}_{3}$ via Catharanthus roseus extract: A novel nanocomposite with high photocatalytic activity," Materials Letters, vol. 277, article 128349, 2020.

[20] S. Saif, A. Tahir, and Y. Chen, "Green synthesis of iron nanoparticles and their environmental applications and implications," Nanomaterials, vol. 6, no. 11, p. 209, 2016.

[21] J. Saini, D. Kashyap, B. Batra, S. Kumar, R. kumar, and D. K. Malik, "Green synthesis of silver nanoparticles by using neem (Azadirachta Indica) and Amla (Phyllanthus Emblica) leaf extract," Indian Journal Of Applied Research, vol. 3, no. 5, pp. 209-210, 2011.

[22] A. Ebrahiminezhad, A. Zare-Hoseinabadi, A. Berenjian, and Y. Ghasemi, "Green synthesis and characterization of zerovalent iron nanoparticles using stinging nettle (Urtica dioica) leaf extract," Green Processing and Synthesis, vol. 6, no. 5, 2017.

[23] Y. Vitta, M. Figueroa, M. Calderon, and C. Ciangherotti, "Synthesis of iron nanoparticles from aqueous extract of Eucalyptus robusta $\mathrm{Sm}$ and evaluation of antioxidant and antimicrobial activity," Materials Science for Energy Technologies, vol. 3, pp. 97-103, 2020.

[24] T. Shahwan, S. Abu Sirriah, M. Nairat et al., "Green synthesis of iron nanoparticles and their application as a Fenton-like catalyst for the degradation of aqueous cationic and anionic dyes," Chemical Engineering Journal, vol. 172, no. 1, pp. 258266, 2011.

[25] Y. Wei, Z. Fang, L. Zheng, L. Tan, and E. P. Tsang, "Green synthesis of Fe nanoparticles using Citrus maxima peels aqueous extracts," Materials Letters, vol. 185, pp. 384-386, 2016.

[26] P. Karpagavinayagam and C. Vedhi, "Green synthesis of iron oxide nanoparticles using Avicennia marina flower extract," Vacuum, vol. 160, pp. 286-292, 2019.

[27] M. Senthil and C. Ramesh, "Biogenic synthesis of $\mathrm{Fe}_{3} \mathrm{O}_{4}$ nanoparticles using Tridax procumbens leaf extract and its antibacterial activity on Pseudomonas aeruginosa," Digest Journal of Nanomaterials \& Biostructures (DJNB), vol. 7, no. 4, 2012.

[28] S. Bishnoi, A. Kumar, and R. Selvaraj, "Facile synthesis of magnetic iron oxide nanoparticles using inedible Cynometra ramiflora fruit extract waste and their photocatalytic degradation of methylene blue dye," Materials Research Bulletin, vol. 97, pp. 121-127, 2018.

[29] M. A. Jadidi Kouhbanani, N. Beheshtkhoo, A. M. Amani et al., "Green synthesis of iron oxide nanoparticles usingArtemisia vulgarisleaf extract and their application as a heterogeneous Fenton-like catalyst for the degradation of methyl orange," Materials Research Express, vol. 5, no. 11, article 115013, 2018.

[30] I. A. Radini, N. Hasan, M. A. Malik, and Z. Khan, "Biosynthesis of iron nanoparticles using Trigonella foenum-graecum seed extract for photocatalytic methyl orange dye degradation and antibacterial applications," Journal of Photochemistry and Photobiology B: Biology, vol. 183, pp. 154-163, 2018. 